\title{
Thermal equation of state of cubic boron nitride: Implications for a high-temperature pressure scale
}

\author{
Alexander F. Goncharov, ${ }^{1,2}$ Jonathan C. Crowhurst, ${ }^{2}$ John K. Dewhurst, ${ }^{3}$ Sangeeta Sharma, ${ }^{4}$ Chrystele Sanloup,${ }^{5}$ \\ Eugene Gregoryanz, ${ }^{1,6}$ Nicolas Guignot, ${ }^{7}$ and Mohamed Mezouar ${ }^{7}$ \\ ${ }^{1}$ Geophysical Laboratory, Carnegie Institution of Washington, 5251 Broad Branch Road NW, Washington, DC 20015, USA \\ ${ }^{2}$ Lawrence Livermore National Laboratory, University of California, 7000 East Avenue, Livermore, California 94551, USA \\ ${ }^{3}$ School of Chemistry, The University of Edinburgh, Edinburgh, United Kingdom \\ ${ }^{4}$ Institut für Theoretische Physik, Freie Universität Berlin, Berlin, Germany \\ and Fritz Haber Institute of the Max Planck Society, Berlin, Germany \\ ${ }^{5}$ Université Pierre et Marie Curie and Insitut de Physique du Globe de Paris, Paris, France \\ ${ }^{6}$ School of Physics and Center for Science at Extreme Conditions, University of Edinburgh, Edinburgh, United Kingdom \\ ${ }^{7}$ European Synchrotron Radiation Facility, Grenoble, France \\ (Received 28 February 2007; published 21 June 2007)
}

\begin{abstract}
The equation of state of cubic boron nitride $(\mathrm{cBN})$ has been determined to a maximum temperature of $3300 \mathrm{~K}$ at a simultaneous static pressure of up to more than $70 \mathrm{GPa}$. Ab initio calculations to $80 \mathrm{GPa}$ and $2000 \mathrm{~K}$ have also been performed. Our experimental data can be reconciled with theoretical results and with the known thermal expansion at 1 bar if we assume a small increase in pressure during heating relative to that measured at ambient temperature. The present data combined with the Raman measurements we presented earlier form the basis of a high-temperature pressure scale that is good to at least $3300 \mathrm{~K}$.
\end{abstract}

DOI: 10.1103/PhysRevB.75.224114 PACS number(s): 62.50. + p, 07.35. +k, 61.10.Nz, 65.40.De

\section{INTRODUCTION}

Knowledge of the thermal equations of state (EOS) of relevant materials is often vital to a variety of disciplines that include geophysics, geochemistry, planetary sciences, and material science. The same is true for the phase boundaries and chemical reactivity under high pressure and simultaneous high temperature. If measured accurately, this information can be used to make conclusions about the phase and chemical composition of the interior of the Earth as well as the interiors of other planets. It can also be used to infer thermodynamic parameters under extreme conditions which in turn are used to test and develop theoretical models.

Of the many experimental challenges that must be surmounted in order to acquire this information one of the most problematic is the need to accurately measure pressure under conditions of simultaneous high temperature and static pressure. The diamond-anvil cell (DAC) is typically heated in one of two ways: external electrical resistive heating, in which the entire sample chamber is uniformly heated, and laser heating in which only the sample or part of the sample is heated. The former technique is used to generate temperatures up to approximately $1000 \mathrm{~K}$ and involves keeping the DAC hot for extended periods of time during which pressure may be adjusted. Naturally, a sensor that is believable under these conditions must be employed. However, while a sensor based on, for example, the ruby or $\mathrm{Sm}: \mathrm{SrB}_{4} \mathrm{O}_{7}$ fluorescence scales may be appropriate for chemically inert samples such as molecular nitrogen or argon they fail in the case of other common materials such as water due to chemical reactivity. In this case other less sensitive sensors must be used [e.g., cBN (Refs. 1 and 2)] or more sophisticated methods adopted (such as the double cavity approach of Refs. 3 and 4). In the laser-heating technique temperatures of up to $\sim 5000 \mathrm{~K}$ may be achieved in much more localized regions. For this method it is often assumed that pressure does not change appreciably at high temperature and that pressure obtained at room temperature (using, e.g., the ruby scale) is sufficiently accurate. Since high temperatures may be obtained in just a few seconds it is practical to adjust pressure only when the sample is cold. However, the pressure may indeed change significantly at high temperature for various reasons, one of which is due to restricted thermal expansion of the sample into a resistive pressure medium-the so-called "thermal pressure."

In the past, the EOS of several materials with simple crystal structures has been used to construct the high-pressure scale at high temperature. Metals such as $\mathrm{Au}, \mathrm{Pt}, \mathrm{W}, \mathrm{Mo}, \mathrm{Pd}$, $\mathrm{Ag}, \mathrm{Al}$, and $\mathrm{Cu}$, and also nonmetallic materials such as $\mathrm{MgO}$ and $\mathrm{NaCl}$, whose equations of state are established based on shock compression experiments and thermodynamic data, are commonly used as pressure standards. ${ }^{5-11}$ The necessary temperature correction of the lattice constants is usually performed on the basis of the Mie-Grüneisen theory. ${ }^{7,11,12}$ As is evident from a number of conflicting data, ${ }^{9,12-17}$ an accurate determination of pressure at high temperature is difficult because of large uncertainty in calculating the volume at isothermal conditions. The necessary corrections require taking into account possible nonlinearity of the volume dependence of the Gruneisen parameter (e.g., Ref. 9), and also a possibly significant electronic contribution to the thermal expansion (e.g., Ref. 18), and are thus associated with large uncertainties. Also, as has been pointed out, ${ }^{5,11}$ the measured Hugoniot stresses of a solid should be corrected for the shear strength in order to predict lattice constants under uniform compression - a situation more appropriate to the DAC environment. Such a procedure naturally leads to an additional uncertainty.

An alternative way of constructing an accurate primary pressure scale at high temperature would be the construction of a so-called redundant scale based on simultaneous density 
and sound velocity measurements ${ }^{8}$ under static conditions. This method requires only a relatively small correction from an adiabatic to an isothermal path, so it is expected to be more accurate, especially in the case where density and sound velocity are determined in truly the same experiment (without the use of a "transfer function" such as the ruby manometer as in Ref. 8). The results of both calibration procedures give comparable results for the room-temperature scale, although more recent studies suggest the necessity of a small correction ${ }^{10,11,19-21}$ consistent with an earlier suggestion $^{22}$ that the ruby scale should be adjusted according to Raman and EOS studies of diamond.

In this paper we present experimental angle-dispersive x-ray-diffraction (XRD) data of cubic boron nitride at high temperatures up to $3300 \mathrm{~K}$ in argon using the laser-heated DAC. We also present the results of our theoretical calculations of the equation of state. Experiment and theory are in good agreement at room temperature, but the experimentally determined thermal expansion at high pressure is smaller than that theoretically computed and furthermore does not agree with the known thermal expansion at 1 bar. ${ }^{23}$ We find, however, that the assumption of a moderate thermal pressure during heating reconciles experiment and theory. Our Raman data obtained under similar conditions (albeit to lower temperatures $)^{2}$ showed a much larger relative shift of frequency (compared to the relative change of the lattice constant) with temperature, so thermal pressure correction was not as critical as in this work. These two sets of high pressure-temperature data are a basis for pressure determination at high temperature.

\section{EXPERIMENT}

We performed our laser heating experiment in a symmetric diamond cell with Boehler-Almax seats, ${ }^{24}$ which allow large angular access for XRD measurements. cBN submicron powder was pressed into a $10-\mu \mathrm{m}$-diameter hole in an iridium square of approximately $40 \times 40 \times 12 \mu \mathrm{m}^{3}$. Argon was loaded cryogenically and served as both the pressure medium and the thermal insulation. XRD measurements were performed at the ID27 beamline of the European Synchrotron Radiation Facility (ESRF). A monochromatic radiation source at $33.2 \mathrm{keV}$ was focused to a spot of $5 \mu \mathrm{m}$ in diameter. Powder-diffraction patterns were collected on a MAR 345 two-dimensional position sensitive detector. A typical collection time was $60 \mathrm{~s}$. The beamline features a dedicated double-sided laser-heating system that incorporates reflective optics in order to minimize chromatic aberrations. This is particularly useful when using thermal emission spectra to infer temperature. Before a high-temperature experimental run (at each pressure), the heating laser spots (of approximately $20 \mu \mathrm{m}$ in diameter) were carefully positioned concentric with the hole in the Ir coupler whose location with respect to the incident $\mathrm{X}$-ray beam was previously selected by optimizing the XRD signal of the cBN sample. During the alignment procedure the temperatures on both sides of the Ir square were measured and were made equal by appropriately distributing the laser beam power. These preliminary heatings also helped to relax stresses in the sample chamber be-

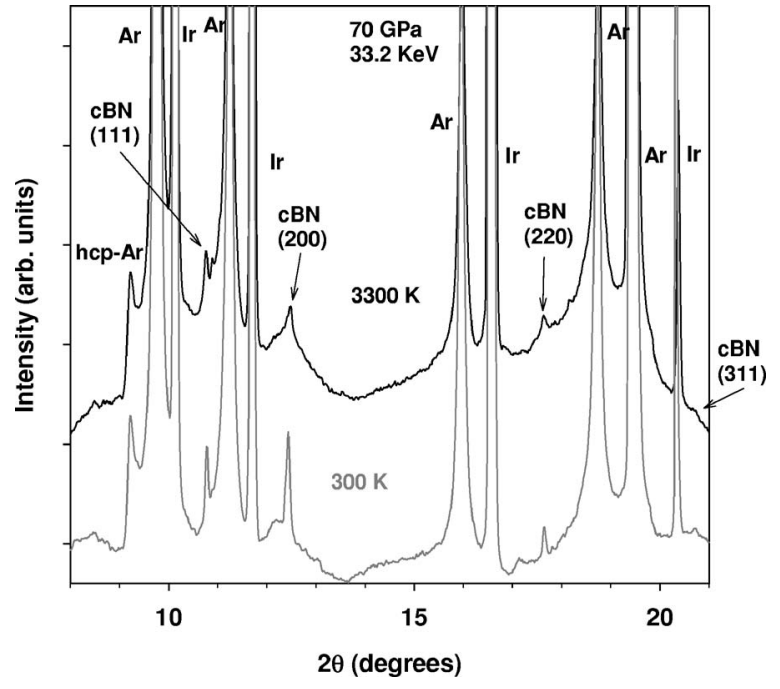

FIG. 1. Representative powder-diffraction patterns showing the effect of temperature on measured diffraction lines at a pressure of $70 \mathrm{GPa}$ (i.e., the nominal pressure measured at room temperature according to the ruby scale).

fore real XRD measurements were made. During the latter, temperatures could only be measured from one side. After each heating, temperature was decreased back to ambient, and an XRD control measurement was made to check the stability of the stress conditions. Pressure was measured before and after the sequence of high-temperature experiments at each pressure point using ruby fluorescence.

Our density-functional theory (DFT) calculations have been described in our previous publication, ${ }^{2}$ where vibrational properties of cBN were presented. Here we concentrate on the thermal equation of state.

\section{RESULTS AND DISCUSSION}

Our experimental diffraction patterns (Fig. 1) show reflections from Ar, Ir, and cBN. Initially, two-dimensional diffraction patterns of $\mathrm{Ar}$ and Ir showed rather uniform rings, which indicated fine randomly oriented crystallites. After a few heatings these rings become spotty, revealing stronger peaks indicative of the formation of larger crystallites. This type of pattern was observed to the highest pressure reached. The cBN diffraction was spotty and did not change substantially. Nevertheless, we always found the lattice constants of cBN determined from different reflections [(111), (200), (311), and sometimes (200) were normally used for refinement of the lattice parameter] were consistent within $0.002 \AA$. The effect of temperature was clearly observed in the shifting of the diffraction lines to larger $d$ spacings and also in their broadening. The lines of all three materials could always be indexed to a cubic structure. Yet above $20 \mathrm{GPa}$ we observed a new ring (Fig. 1), which indicated a partial transformation of Ar to the hep structure. ${ }^{25}$

The EOS of $\mathrm{cBN}$ at room temperature (Fig. 2) has been studied previously by several groups both experimentally (up to $120 \mathrm{GPa}),{ }^{26,27}$ and theoretically. ${ }^{27,28}$ Our experimental data are in excellent agreement with previous experiments 


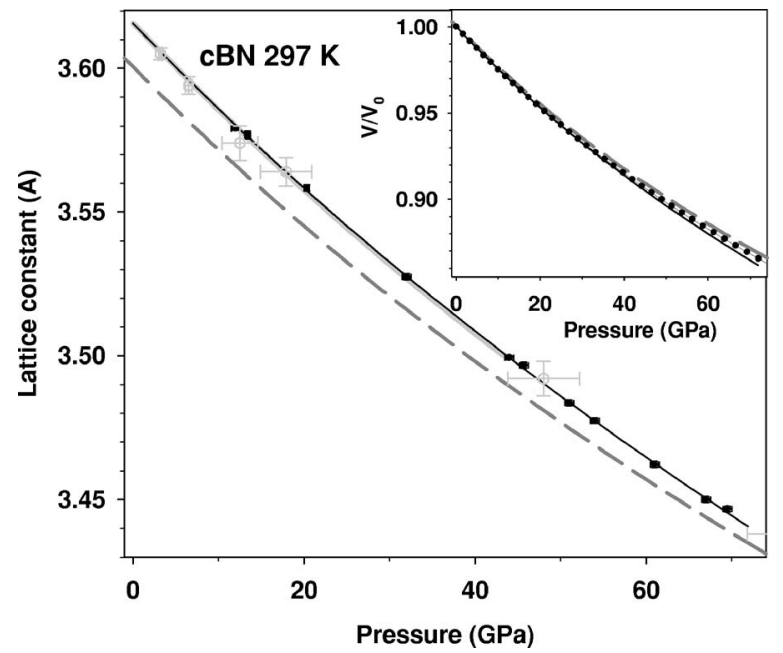

FIG. 2. The room-temperature equation of state of $\mathrm{cBN}$. Solid small points are our experimental x-ray-diffraction data; the thin solid line is the best fit to the Birch-Murnagan EOS. Thick gray line is the best fit to the experimental data from Ref. 26; gray symbols with error bars are from the experimental study of Ref. 27. Dark gray dashed line represents the results of our DFT calculations. The inset shows the comparison of our experiment and theory for reduced volumes. Solid line is experimental data obtained using the ruby scale of Ref. 6 , solid gray line and filled circles: same using a newly proposed ruby calibration (Ref. 11).

(Fig. 2). Moreover, the isothermal bulk modulus $K_{T}$ and its pressure derivative $K_{T}^{\prime}$ at ambient conditions determined from our experiments (Table I) can be compared reasonably well to the adiabatic bulk modulus, $K_{S}=372.3 \pm 3.7 \mathrm{GPa}$ and its derivative $K_{S}^{\prime}=3.48 \pm 0.41$ obtained using ultrasonic interferometry. ${ }^{29}$ The parameters of the theoretically computed EOS are close to the experimentally determined ones (Table I) except for the pressure derivative of the bulk modulus $B^{\prime}$ which is some $17 \%$ larger. Small deviations in the lattice parameter and the bulk modulus are within the usual accuracy of the DFT calculations and we note the typical tendency of the local-density approximation (LDA) to overbind solids.

The deviation of the theory and experiment in $K_{T}^{\prime}$ (see also Ref. 26) is outside the error bars and may be attributed to a systematic underestimation of pressure by the current

TABLE I. Comparison of our experimental and theoretical parameters of the EOS of $\mathrm{cBN}$ at room temperature. The third-order Burch-Murnagan form of the $\operatorname{EOS}\left(P=1.5 K_{T, 0}\left[\left(V_{T, 0} / V\right)^{7 / 3}\right.\right.$ $\left.-\left(V_{T, 0} / V\right)^{5 / 3}\right]\left\{1-0.75\left(4-K_{T, 0}^{\prime}\right)\left[\left(V_{T 0} / V\right)^{2 / 3}-1\right]\right\}$ was used in both cases.

\begin{tabular}{lccc}
\hline \hline EOS & $\begin{array}{c}\text { Lattice } \\
\text { parameter } \\
a_{300,0} \\
(\AA)\end{array}$ & $\begin{array}{c}\text { Bulk } \\
\text { modulus } \\
K_{300,0} \\
(\mathrm{GPa})\end{array}$ & $\begin{array}{c}\text { Pressure } \\
\text { derivative } \\
\text { of } B_{0} \\
K_{300,0}^{\prime}\end{array}$ \\
\hline Experiment & $3.6157(8)$ & $387(4)$ & $3.06(15)$ \\
Theory & 3.6005 & 395.9 & 3.58 \\
Deviation & $-0.4 \%$ & $+2.3 \%$ & $+17 \%$ \\
\hline \hline
\end{tabular}

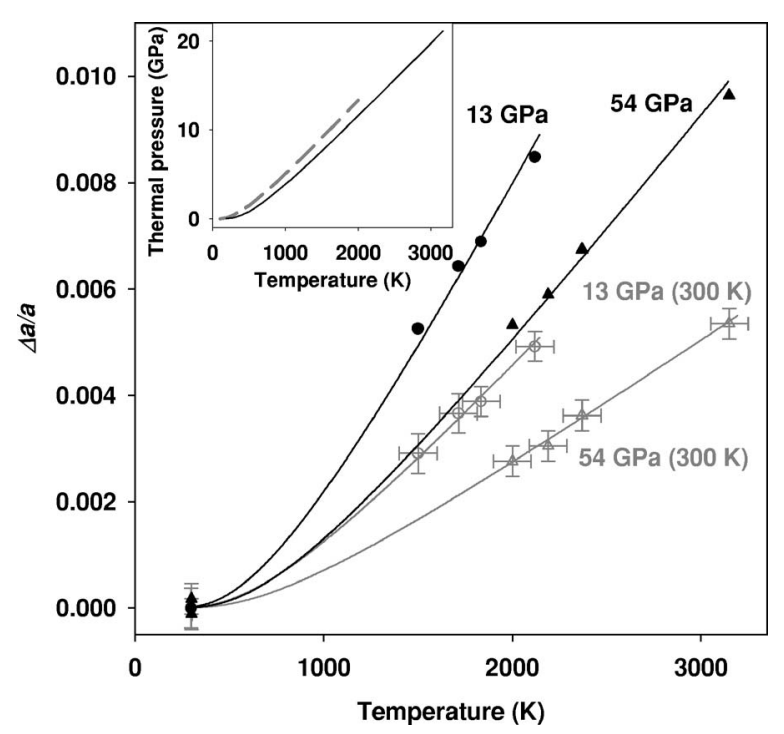

FIG. 3. Temperature dependence of the lattice constant elongation of $\mathrm{cBN}$ at high temperatures. Open symbols: our raw data; filled symbols: corrected to the thermal pressure data (see text). Lines are fits to the experimental data using the Einstein model (see text). The inset shows the thermal pressure calculated under the assumption of a constant $\beta K_{\mathrm{T}}$ product (solid line) in comparison with the theoretical calculations at $0 \mathrm{GPa}$ (dashed gray line).

ruby scale. ${ }^{10,11,19-21}$ If one uses the corrected ruby scale (e.g., Ref. 11, other suggestions are very similar), the agreement between experiment and theory is improved (inset Fig. 2), with the remaining small discrepancy due to the slightly overestimated value of the calculated bulk modulus. The proposed correction ${ }^{10,11,19-21}$ to the most commonly used ruby scale $^{6}$ is about $3 \%$ at $60 \mathrm{GPa}$. We will continue using the room-temperature ruby scale ${ }^{6}$ throughout this paper for consistency. Our results (mainly concerning temperature effects) can easily be adjusted if some other room-temperature pressure scale is assumed.

Our experimental thermal-expansion data show a monotonic increase of lattice parameter with temperature (Fig. 3). Both the Debye and Einstein models are expected to well describe the thermal expansion in $\mathrm{cBN}^{30}$ For simplicity (a simple analytical formula can be used), we determined the linear thermal-expansion coefficient $\beta=d(\ln a) / d T$ by fitting the experimental data to the Einstein model $a=a_{0}\{1$ $\left.+\beta_{\mathrm{hT}} T_{\Theta} /\left[\exp \left(\Theta_{\mathrm{E}} / T\right)-1\right]\right\}$, where $\Theta_{E}$ is the Einstein temperature and $\beta_{\mathrm{hT}}$ is the thermal-expansion coefficient in the limit of high temperature $\left(T \gg \Theta_{\mathrm{E}}, \Theta_{\mathrm{E}}=1900 \mathrm{~K}\right.$ at $\left.1 \mathrm{bar}^{23}\right)$ The experimental data can be described well by this model (Fig. 3) under the assumption of a constant pressure during laser heating. However, the thermal-expansion coefficient (Fig. 4) determined by this procedure at high pressure is too small compared with the theoretical value and is also too small compared with the known 1-bar value. ${ }^{23}$ We conclude that even in the case of the relatively soft argon medium there is a significant thermal pressure. The magnitude of this effect depends critically on the thermal expansivities and bulk moduli of the sample and its surroundings, on their geometry and dimensions, and on the temperature distribution. ${ }^{31,32}$ In this paper we assume for simplicity that 


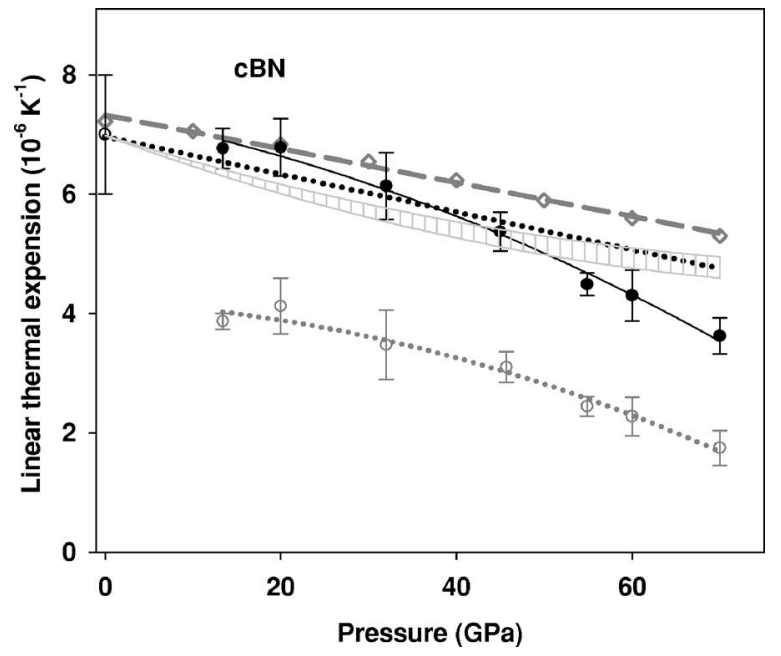

FIG. 4. Pressure dependence of the linear thermal expansion of $\mathrm{cBN}$ in the limit of high temperature $\left(\beta_{\mathrm{hT}}\right)$. Gray open circles and gray dotted line: obtained from the raw data. Filled circles and solid line: obtained from the data corrected for the thermal pressure. Open circle at ambient pressure is from Ref. 23. Gray thick diamonds and gray dashed line: our theoretical calculations. Hatched area and black dotted line correspond to the results of the model calculations, which assume $\gamma=\gamma_{0}(V / V o)^{q}(1 \leqslant q \leqslant 1)$ and $\beta K_{\mathrm{T}}$ $=$ const, respectively (see text for details).

this correction is equal to $30 \%$ of the maximum isochoric value $\int_{\text {Tamb }}^{\text {Texp }} 3 \beta K_{T} d T$ (i.e., when the sample is in an infinitely rigid medium; cubic symmetry of the sample is implied in the case of cBN). This fraction is derived from model calculations for conditions similar to ours. ${ }^{31}$ To estimate the maximum isochoric value we in principle need the pressure dependence of $\beta$ and $K_{\mathrm{T}}$. We began by assuming that the product $\beta K_{\mathrm{T}}$ was pressure independent (e.g., Ref. 33) as we also assumed in our previous paper, ${ }^{2}$ so it can be calculated using 1 bar data (inset to Fig. 3). In the second model we calculated the thermal pressure using a quasiharmonic Debye model (see, e.g., Ref. 9) assuming that the mode Gruneisen parameter depends on volume as $\gamma=\gamma_{0}\left(V / V_{o}\right)^{q},{ }^{34}$ where $\gamma_{0}$ $=1.05$ was chosen to match the thermal expansion at ambient pressure and where $0 \leqslant q \leqslant 1$ was chosen to be in the range of the experimental observations. ${ }^{26,27}$ In this case the thermal pressure is slightly pressure dependent $(<12 \%$ change in the pressure range studied). We stress that our model results for $\beta$ (Fig. 4) do not critically depend on the functional form of the volume dependences of $\gamma$ and the strength of this dependence (determined by $q$ ), because at the highest pressure studied $(70 \mathrm{GPa})$ we are still in the low compression limit $(P \ll K$ for all $T)$. It is interesting that our model calculations agree well to each other, and also reasonably well with our theoretical calculations (Figs. 3 and 4)

After the correction of our high-temperature data as described above, our thermal-expansion data (Fig. 3) can still be fitted appropriately by the Einstein model, and the thermal-expansion coefficients agree well (Fig. 4) in the limit of low pressures with the ambient pressure data. ${ }^{23}$ Our experimental data also agree with our theoretically computed thermal-expansion coefficients up to approximately $30 \mathrm{GPa}$ (Figs. 3 and 4). Our high $P, T$ EOS can be presented in the Birch-Murnagan form

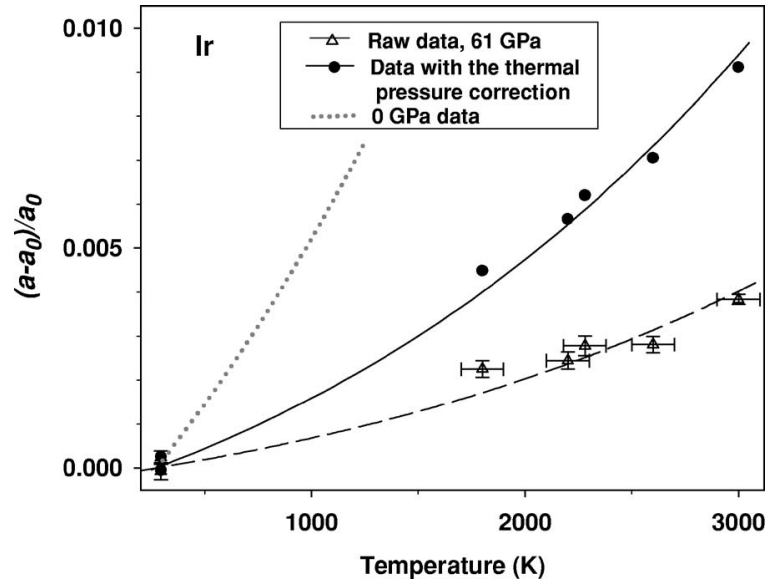

FIG. 5. Temperature dependence of the lattice constant elongation of Ir. Open symbols: our raw data; filled symbols: corrected to the thermal pressure data (see text). Lines are fits to the data using the polynomial form of Ref. 36. Dotted gray line represents the 0 GPa data from Ref. 36.

$$
\begin{aligned}
P= & 1.5 K_{T, 0}\left[\left(V_{T, 0} / V\right)^{7 / 3}-\left(V_{T, 0} / V\right)^{5 / 3}\right]\left\{1-0.75\left(4-K_{T, 0}^{\prime}\right)\right. \\
& \left.\times\left[\left(V_{T 0} / V\right)^{2 / 3}-1\right]\right\}
\end{aligned}
$$

with the temperature-dependent parameters $K_{T, 0}$ $=389.2-7.30 \times 10^{-3} \mathrm{~T}-7.60 \times 10^{-7} \mathrm{~T}^{2} ; \quad K_{T, 0}^{\prime}=3.22-4.47$ $\times 10^{-4} \mathrm{~T} ; \quad a_{T, 0}=3.6140+2.309 \times 10^{-6} \mathrm{~T}+9.831 \times 10^{-9} \mathrm{~T}^{2}$ $-1.35 \times 10^{-12} \mathrm{~T}^{3}$. A faster decrease of the experimental $\beta$ with pressure compared to the theoretically computed one (Fig. 4) must be treated with caution since it depends on the rather crude assumption of a thermal pressure that is a constant fraction of the maximum thermal pressure (see above). Since the geometrical and thermoelastic parameters of the materials in the sample cavity change significantly with compression, the latter conjecture may not hold.

To further test this assumption we analyzed the thermalexpansion data of Ir that we simultaneously acquired (Fig. 1). We find that the room-temperature EOS of Ir is in good agreement with the literature data; ${ }^{35}$ a Birch-Murnagan EOS yields parameters $B_{0}=391(4)$ and $B^{\prime}=3.3(2)$ which may be compared to $B_{0}=383(14) \mathrm{GPa}$ and $B^{\prime}=3.1(8)$. The thermalexpansivity data of Ir at ambient pressure cannot be approximated by the Debye or Einstein model, because the thermalexpansion coefficient exhibits a monotonic increase with temperature well above the Debye temperature ${ }^{36,37}$ unlike the case of cBN (see above). This behavior is likely due to an electronic contribution to the thermal conductivity in the case of metallic Ir (this in turn results in a nonlinear contribution to the thermal expansion). We approximated our data (Fig. 5) using the same polynomial as in Ref. 37. We found that the thermal-expansion coefficient extrapolated to $1 \mathrm{bar}$ was, as for $\mathrm{cBN}$, underestimated with respect to the known value (Fig. 6): $4 \times 10^{-6} \mathrm{~K}^{-1}$ compared with $6.13 \times 10^{-6} \mathrm{~K}^{-1}$. If we assume the same (pressure independent) thermal pressure as we did for $\mathrm{cBN}$ we obtain much better agreement (Fig. 6). We conclude that the assumption of a moderate thermal pressure ${ }^{31}$ allows us to interpret our data selfconsistently. No attempt has been made to rationalize the 


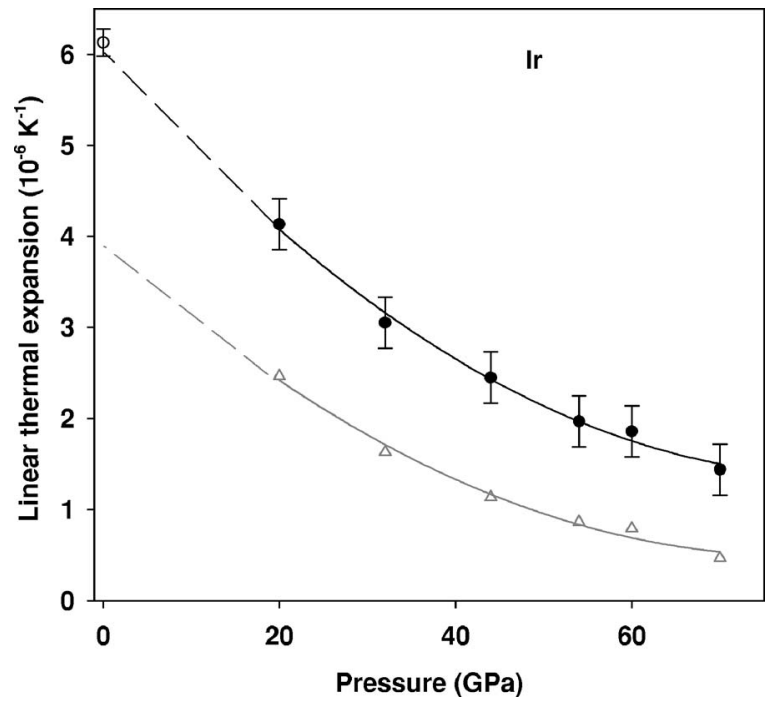

FIG. 6. Pressure dependence of the linear thermal-expansion coefficient of Ir at room temperature. Gray open triangles and gray line: obtained from the raw data. Filled circles and solid line: obtained from the data corrected to the thermal pressure. Open circle at ambient pressure is from Ref. 36 .

thermal-expansion data of Ar because of large temperature gradients through this material during heating. We notice, though, that the EOS of Ar at room temperature is consistent with previous observations. ${ }^{38}$

The results presented here show that $\mathrm{cBN}$, even in minute amounts, can serve as an excellent pressure sensor even in the realm of laser-heating temperatures. In previous combined laser-heating XRD experiments Pt has typically been used as a pressure sensor (see, e.g., Refs. 39 and 40). It is chemically stable, and its thermal equation of state is believed to have been determined with sufficient accuracy. ${ }^{16,41,42} \mathrm{Au}$ is also chemically stable, but there is no consensus concerning the thermal EOS of this material. ${ }^{41,43-45}$ Other commonly used high-temperature pressure sensors such as $\mathrm{NaCl}$ and $\mathrm{MgO}$ are difficult to use in laser heating experiments because of the requirement of keeping the sample and the gauge in intimate thermal contact. These materials have relatively low thermal conductivities, so it is difficult to avoid large temperature gradients.

We argue that $\mathrm{cBN}$ has a number of advantages compared to $\mathrm{Pt}$ and these other materials. The melting temperature of $\mathrm{cBN}$ is higher than any of them (above a pressure of $9 \mathrm{GPa}$ it exceeds $3500 \mathrm{~K}) .{ }^{46}$ For comparison, the melting point of $\mathrm{Pt}$ is about $2800 \mathrm{~K}$ at $40 \mathrm{GPa}{ }^{47} \mathrm{cBN}$ also has a very high thermal conductivity (approaching that of diamond, its structural and electronic analog), which makes it equally useful for laser heating experiments as $\mathrm{Pt}$ and other metals. One important advantage of cBN compared to metallic sensors is its optical transparency which allows Raman and Brillouin scattering measurements. This property has been exploited to construct an optical pressure scale based on Raman measure- ments to at least $2200 \mathrm{~K} .^{2}$ Potential redundant measurements (as described above ${ }^{8}$ ) of elasticity using Brillouin scattering together with measurements of density using XRD (Ref. 48) on the same sample under the same conditions should also be possible.

We believe that even in the absence of a comprehensive calibration procedure, the data presented can be used for in situ pressure determination with accuracy comparable to or even exceeding that of previous scales. The advantage of using cBN is simplicity of sample preparation and an ability to use this material in both optical and synchrotron $\mathrm{x}$-ray investigations combined with its high melting temperature and chemical stability (see above). With the development of pulsed Raman techniques, ${ }^{49,50}$ Raman measurements to $\sim 5000 \mathrm{~K}$ (depending on the wavelength of the pulsed laser source) should become feasible and thus also pressure determination (if the $\mathrm{cBN}$ remains solid). The Raman data of $\mathrm{cBN}$ obtained to $2000 \mathrm{~K}$ and $40 \mathrm{GPa}$ (Ref. 2) require a small correction since the thermal pressure was assumed to be zero in the case of the argon medium used in that work. On the basis of the present data (assuming the same thermal pressure), we suggest the following corrected expression for a pressure scale at high temperatures:

$$
P=(R / b)\left[\left(\nu / \nu_{\mathrm{o}}\right)^{b}-1\right],
$$

where $\nu$ is the Raman frequency, $R, \nu_{0}$, and $b$ are temperature-dependent parameters: $R=325.6-1.11 \times 10^{-2} \mathrm{~T}$ $-8.90 \times 10^{-6} \mathrm{~T}^{2}, \quad \nu_{\mathrm{o}}=1060.2-0.012 \mathrm{~T}-1.57 \times 10^{-5} \mathrm{~T}^{2}, \quad b$ $=3.48-1.75 \times 10^{-4} \mathrm{~T}-8.55 \times 10^{-8} \mathrm{~T}^{2}$.

In conclusion, we have presented the results of the first $x$-ray-diffraction measurements of the thermal EOS of cBN up to $3300 \mathrm{~K}$ and $70 \mathrm{GPa}$ and theoretical calculations to $2000 \mathrm{~K}$ and $80 \mathrm{GPa}$. The experimental EOS can be well constrained using a Mie-Gruneisen approximation or approximate empirical relations. This work thus establishes highpressure pressure scale at temperatures exceeding $3000 \mathrm{~K}$.

First-principles theoretical calculations reproduce the major features of the thermal EOS at high temperature. A small initial offset and a difference in curvature at high pressure can be imputed to the usual uncertainty of the equilibrium volume in the DFT calculations and to the necessity to further rectify the high-pressure ruby scale, respectively. The deviation between theory and experiment at high temperatures is due to an incomplete description of the anharmonicity in the long-wavelength modes and not because of the limited accuracy of the LDA. These finite $q$ modes were approximated with a Debye-like model.

\section{ACKNOWLEDGMENTS}

This work was performed under the auspices of the U. S. Department of Energy by the University of California, Lawrence Livermore National Laboratory under Contract No. W-7405-Eng-48. We acknowledge the European Synchrotron Radiation Facility for provision of synchrotron radiation facilities. 
${ }^{1}$ F. Datchi and B. Canny, Phys. Rev. B 69, 144106 (2004).

${ }^{2}$ A. F. Goncharov, J. C. Crowhurst, J. K. Dewhurst, and S. Sharma, Phys. Rev. B 72, 100104(R) (2005).

${ }^{3}$ F. Datchi, P. Loubeyre, and R. LeToullec, Phys. Rev. B 61, 6535 (2000).

${ }^{4}$ E. H. Abramson and J. M. Brown, Geochim. Cosmochim. Acta 68, 1827 (2004).

${ }^{5}$ H. K. Mao, P. M. Bell, J. W. Shaner, and D. J. Steinberg, J. Appl. Phys. 49, 3276 (1978).

${ }^{6}$ H. Mao, J. Xu, and P. Bell, J. Geophys. Res. 91, 4673 (1986).

7 J. M. Brown, J. Appl. Phys. 86, 5801 (1999).

${ }^{8}$ C.-S. Zha, H.-K. Mao, and R. J. Hemley, Proc. Natl. Acad. Sci. U.S.A. 97, 13494 (2000).

${ }^{9}$ S. Speziale, C.-S. Zha, T. S. Duffy, R. J. Hemley, and H. K. Mao, J. Geophys. Res. 106, 515 (2001).

${ }^{10}$ A. Dewaele, P. Loubeyre, and M. Mezouar, Phys. Rev. B 70, 094112 (2004).

${ }^{11}$ A. D. Chijioke, W. J. Nellis, A. Soldatov, and I. F. Silvera, J. Appl. Phys. 98, 114905 (2005).

${ }^{12}$ O. L. Anderson, D. G. Isaak, and S. Yamamoto, J. Appl. Phys. 65, 1534 (1989).

${ }^{13}$ T. Irifune, N. Nishiyama, K. Kuroda, T. Inoue, M. Isshiki, W. Utsumi, K. Funakoshi, S. Urakawa, T. Uchida, T. Katsura, and O. Osamu, Science 279, 1698 (1998).

${ }^{14}$ K. Hirose, Y. Fei, S. Ono, T. Yagi, and K. Funakoshi, Earth Planet. Sci. Lett. 184, 567 (2001).

${ }^{15}$ S.-H. Shim, T. S. Duffy, and G. Shen, Nature (London) 411, 571 (2001).

${ }^{16}$ Y. Fei, J. Li, K. Hirose, W. Minarik, J. Van Orman, C. Sanloup, W. van Westrenen, T. Komabayashi, and K. Funakoshi, Phys. Earth Planet. Inter. 143/144, 516 (2004).

${ }^{17}$ S.-H. Shim, T. S. Duffy, and T. Kenichi, Earth Planet. Sci. Lett. 203, 729 (2002).

${ }^{18}$ M. Matsui and N. Shima, J. Appl. Phys. 93, 9679 (2003).

${ }^{19}$ W. B. Holzapfel, J. Appl. Phys. 93, 1813 (2003).

${ }^{20}$ P. I. Dorogokupets and A. R. Oganov, Dokl. Akad. Nauk 391, 515 (2003) [Doklady Earth Sciences 391A, 854 (2003)].

${ }^{21}$ K. Kunc, I. Loa, and K. Syassen, Phys. Rev. B 68, 094107 (2003).

${ }^{22}$ I. V. Aleksandrov, A. F. Goncharov, A. N. Zisman, and S. M. Stishov, Zh. Eksp. Teor. Fiz. 93, 680 (1987) [Sov. Phys. JETP 66, 384 (1987)].

${ }^{23}$ G. A. Slack and S. F. Bertram, J. Appl. Phys. 46, 89 (1975).

${ }^{24}$ R. Boehler and K. De Hantsetters, High Press. Res. 24, 391 (2004)

${ }^{25}$ D. Errandonea, R. Boehler, S. Japel, M. Mezouar, and L. R. Benedetti, Phys. Rev. B 73, 092106 (2006).

${ }^{26}$ E. V. Jakovenko, I. V. Aleksandrov, A. F. Goncharov, and S. M. Stishov, Zh. Eksp. Teor. Fiz. 95, 2097 (1989) [Sov. Phys. JETP 68, 1213 (1989)].
${ }^{27}$ E. Knittle, R. Wentzcovitch, R. Jeanloz, and M. Cohen, Nature (London) 337, 349 (1989).

${ }^{28}$ R. M. Wentzcovitch, M. L. Cohen, and P. K. Lam, Phys. Rev. B 36, 6058 (1987).

${ }^{29}$ M. H. Manghnani in Proceedings of the 5th NIRIM International Symposium on Advanced Material (ISAM '98), Tsukuba, Japan, March 1-5, 1998, p. 73.

${ }^{30}$ C. Roder, S. Einfeldt, S. Figge, and D. Hommel, Phys. Rev. B 72, 085218 (2005).

${ }^{31}$ A. Dewaele, G. Fiquet, and Ph. Gillet, Rev. Sci. Instrum. 69, 2421 (1998).

${ }^{32}$ D. L. Heinz, Geophys. Res. Lett. 17, 1161 (1990).

${ }^{33}$ O. L. Anderson, K. Masuda, and D. G. Isaak, Phys. Earth Planet. Inter. 91, 3 (1995).

${ }^{34}$ J.-P. Poirier and A. Tarantola, Phys. Earth Planet. Inter. 109, 1 (1998).

${ }^{35}$ H. Cynn, J. E. Klepeis, C.-S. Yoo, and D. A. Young, Phys. Rev. Lett. 88, 135701 (2002).

${ }^{36}$ H. P. Singh, Acta Crystallogr., Sect. A: Cryst. Phys., Diffr., Theor. Gen. Crystallogr. A24, 469 (1968).

${ }^{37}$ J. J. Halvorson and R. T. Wimber, J. Appl. Phys. 43, 2519 (1972).

${ }^{38}$ M. Ross, H. K. Mao, P. M. Bell, and J. A. Xu, J. Chem. Phys. 85, 1028 (1986).

${ }^{39}$ G. Fiquet, D. Andrault, A. Dewaele, T. Charpin, M. Kunz, and D. Haüsermann, Phys. Earth Planet. Inter. 105, 21 (1998).

${ }^{40}$ S.-H. Shim, T. S. Duffy, and G. Shen, J. Geophys. Res. 105, 25955 (2000).

${ }^{41}$ J. C. Jamieson, J. N. Fritz, and M. H. Manghnani, in High Pressure Research in Geophysics, edited by S. Akimoto and M. H. Manghnani (Center for Academic Publications, Tokyo, 1982), p. 27.

${ }^{42}$ N. C. Holmes, J. A. Moriarty, G. R. Gathers, and W. J. Nellis, J. Appl. Phys. 66, 2962 (1989).

${ }^{43}$ D. L. Heinz and R. Jeanloz, J. Appl. Phys. 55, 885 (1984).

${ }^{44}$ O. L. Anderson, D. G. Isaak, and S. Yamamoto, J. Appl. Phys. 65, 1534 (1989).

${ }^{45}$ S. Shim, T. S. Duffy, and K. Takemura, Earth Planet. Sci. Lett. 203, 729 (2002).

${ }^{46}$ F. P. Bundy and R. H. Wentorf, Jr., J. Chem. Phys. 38, 1144 (1963); F. R. Corrigan and F. P. Bundy, ibid. 63, 3812 (1975).

${ }^{47}$ A. Kavner and R. Jeanloz, J. Appl. Phys. 83, 7553 (1998).

${ }^{48}$ S. V. Sinogeikin, J. D. Bass, V. Prakapenka, D. Lakshtanov, G. Shen, C. Sanchez Valle, and M. Rivers, Rev. Sci. Instrum. 77, 103905 (1998).

${ }^{49}$ A. F. Goncharov and J. Crowhurst, Rev. Sci. Instrum. 76, 063905 (2005).

${ }^{50}$ S. Shim, R. Lamm, S. Rekhi, K. Catalli, J. Santillan, and S. Lundin, EOS Trans. Am. Geophys. Union 86(52), Fall Meeting Supplement, Abstract MR13B-02, F999 (2005). 